\title{
Siögren's Syndrome and The Respiratory System: A Comprehensive Approach
}

\author{
Fatima Mahmood ${ }^{1 *}$, Moiz Ehtesham', Syed Muhammad Mashhood Ali Bokhari², Ruben A. Peredo-Wende ${ }^{3}$ \\ 'Department of Internal Medicine, Albany Medical College, Albany, NY, USA \\ ${ }^{2}$ University of Texas Southwestern Medical Center, Dallas, TX, USA \\ ${ }^{3}$ Rheumatology Stratton Veteran Medical Center, Albany, NY, USA
}

Article Info

\section{Article Notes}

Received: September 06, 2021

Accepted: December 01, 2021

\section{${ }^{*}$ Correspondence:}

Dr. Fatima Mahmood, Department of Internal Medicine, Albany Medical College, Albany, NY, USA; Email: mahmoof@amc.edu

() 2021 Mahmood F. This article is distributed under the terms of the Creative Commons Attribution 4.0 International License.

\section{Introduction}

Sjögren's syndrome (SS) is an autoimmune connective tissue disease characterized by lymphocytic infiltration of exocrine glands leading to progressive inflammation and destruction. This condition mainly involves the lacrimal and salivary glands and is associated with development of the classic dry eyes and mouth ${ }^{1}$. Additionally, an estimated third of the population develops extra-glandular manifestations involving the cutaneous, cardiovascular, neurologic, renal, pulmonary, and musculoskeletal system ${ }^{2,3}$.

SS can present as a single autoimmune disorder, primary Sjögren's syndrome (pSS), or it can occur in conjunction with other autoimmune conditions, secondary Sjögren's syndrome (sSS) ${ }^{4}$. The 2016 American College of Rheumatology/European League Against Rheumatism classification criteria for pSS includes presence of ocular or oral dryness and a score of $\geq 4$ from criteria which is described in Table 1. This criterion is not applicable to patients who are receiving anti-cholinergic medications or have history of head and neck radiation, hepatitis $\mathrm{C}$ infection, acquired immunodeficiency syndrome (AIDS), sarcoidosis, amyloidosis, graft versus host disease (GVHD) or IgG4-related disease ${ }^{5}$.

As per studies, a fifth of patient with SS develop pulmonary manifestations which are often associated with poor clinical outcomes $^{6}$. In a study involving 37 patients with pSS and normal chest x-ray results, abnormal radiographic signs were present in 24 patients $(65 \%)$ on a high-resolution chest CT (HRCT). This percentage represents patients who were asymptomatic at the time the CT scan was performed ${ }^{7}$. Patients with SS who develop interstitial lung disease (ILD) have a poor survival rate of roughly twofold compared to those without ILD. Many of the respiratory symptoms may associate with a particular component of the respiratory tree especially small airways ${ }^{9}$. Therefore, complete evaluation in patients with pSS or sSS must include chest imaging and pulmonary function testing.

Table 1: Criteria items for diagnosis of $\mathrm{pSS}$ based on ACR/EULAR

\begin{tabular}{|l|c|}
\hline \multicolumn{1}{|c|}{ Criteria } & Score \\
\hline Positive Anti SSA/Ro levels & 3 \\
\hline $\begin{array}{l}\text { Labial salivary gland with focal lymphocytic sialadenitis, focus score } \\
\geq 1 \text { foci/4 mm2 }\end{array}$ & 3 \\
\hline Ocular staining score $\geq 5$ in at least 1 eye & 1 \\
\hline Schirmer's test $\leq 5 \mathrm{~mm} / 5$ minutes in at least 1 eye & 1 \\
\hline Unstimulated whole saliva flow rate $\leq 0.1 \mathrm{ml} /$ minute & 1 \\
\hline
\end{tabular}


Pulmonary manifestation of SS can be divided based on its effect on the upper respiratory tract (ears, nose, mouth, sinuses, and trachea), lower respiratory tract (bronchi and bronchioles), lung parenchyma and, rarely, the pleura.

- Upper airway disease is characterized by invasion of lymphocytic cells with associated dysmotility of nasal and tracheobronchial cilia ${ }^{10,11}$. This leads to a pro-inflammatory state manifesting as cough, dysphagia, or hoarseness ${ }^{12}$. Additionally, aural and nasal pruritus, crusting, mucosal ulceration, and epistaxis are also present ${ }^{13,14}$. Chronic inflammation causes development of xerotrachea, xerobronchitis and/or bronchiectasis ${ }^{15,16}$. Overall, patients develop hypersensitivity to environmental allergens such as smoke, dust, and other environmental hazards. Compared to allergic bronchial asthma, pulmonary SS is characterized by an exaggerated response to the methacholine challenge test and cellular activity, composed of mast cell, neutrophils, and lymphocytes, as opposed to eosinophilic infiltration seen in allergic asthma $^{17,18}$. Oftentimes patients with SS may be misclassified as having bronchial asthma, especially in women between 40-50 years of age ${ }^{9}$.

- Bronchioles are often involved, and the most common presentation is follicular bronchiolitis, in which an inflammatory state drives the bronchial epithelium to form lymphocytic aggregates along the epithelium ${ }^{19}$. Other types of bronchiolitis documented in SS include chronic, obliterative, lymphocytic, constrictivedestructive, and pan-bronchiolitis ${ }^{10,13}$. Chronic bronchiolitis appears as centrilobular abnormalities on HRCT ${ }^{20,21}$.

- Involvement of the lung parenchyma is associated with a 5-year mortality risk of $\sim 20 \%{ }^{22}$. Most identified pattern in interstitial lung disease (ILD) is non-specific interstitial pneumonia (NSIP); others include organizing pneumonia (OP), usual interstitial pneumonia (UIP), lymphocytic interstitial pneumonia (LIP), and diffuse interstitial amyloidosis ${ }^{10,13}$. ILD can often be the initial presentation in one-third of patients with SS and often presents at an older age, prolonged disease duration and with concurrent dry eyes, mouth, and neuropathic symptoms ${ }^{10,23}$. When the respiratory system is affected, pulmonary function tests including FEV, FVC and DLCO often indicate a restrictive pattern. Associated serology presenting with ILD includes anti-SSA/Ro antibodies, ANA, RF and anti-CCP (weakly positive) ${ }^{10,24}$. Imaging including a chest radiograph followed by an HRCT to identify reticular opacities, parenchymal disease and bronchiectasis are of great utility. Furthermore, bronchoalveolar lavage also reveals lymphocyte infiltration ${ }^{5}$.

\section{Pathogenesis and mechanisms}

The glandular destruction in SS is secondary to lymphocytic and other monocytic inflammatory cell infiltration. Histopathology obtained from lacrimal and salivary glands in patients is consistent with grouping of inflammatory cells around glandular ducts. Similarly, infiltration of inflammatory cells is also observed adjacent to epithelial cells in extra-glandular tissues ${ }^{25}$. This gives rise to the concept of a similar pathogenic autoimmune process affecting the glands as well as the epithelium in extra-glandular organs eliciting a cellular and humoral immune response, termed as autoimmune epitheliitis ${ }^{26,27}$. Furthermore, studies suggest that the increased lymphocytic focus score of salivary gland involvement is associated with increased prevalence of pulmonary $\mathrm{SS}^{28}$.

This autoimmune process is triggered by a complex interaction of cellular and environmental factors involving the CD4+ T cells, CD8+ T cells, T regulatory cells, B cells, interleukins and interferons ${ }^{29}$. Studies have defined activation of $\mathrm{B}$ and $\mathrm{T}$ cells in response to common antigens as well as muscarinic receptors expressed on epithelial cells. The resulting compensatory increase in expression of muscarinic receptors leads to hyper-cholinergic response seen in respiratory airways ${ }^{30}$.

Another proposed mechanism is the release of autoantigens from destruction of salivary glands resulting in stimulation of Toll-like receptors (TLRs) on antigen presenting cells (APCs) that in turn increases the production of interferons and interleukins firing a positive feedback response to stimulate $\mathrm{B}$-cells and subsequently increase auto-antibody production ${ }^{31}$. Particularly, type 1 IFN, IL-12, CD4+ T cells and IFN-gamma mediate tissue damage. The role of IL-12 has been demonstrated through transgenic mouse models demonstrating IL-12 expression in lung tissues. In a study these transgenic mice were evaluated for development of pulmonary abnormalities. At 4 months they were found to develop airway epithelium associated lymphocytic and alveolar macrophages infiltrates and overall increased cell proliferation that ultimately led to impaired ciliary clearance. These findings bear strong resemblance to patients with pulmonary SS, suggesting the potential role of IL-12 in the inflammatory process ${ }^{32}$.

Other cellular mediators triggered by TLR stimulation are release of pro-inflammatory IL-17 and CD4+ cells (Th1 and Th17). The role of IL-17 is well-established in pathogenesis of chronic inflammatory and autoimmune diseases $^{33}$. Binding of IL-17 to its receptor is implicated in the transcription of inflammatory genes inducing production of cytokines (IL-1, IL-6, TNF), matrix metalloproteinases and chemokines (CXCL, CCL and IL-8) ${ }^{34}$. Th1 and Th17 cells cause increased production of IFN-gamma, which is implicated in increased apoptosis, tissue damage, and promotion of $\mathrm{B}$ cell production, differentiation and 
formation of germinal centers that are characteristically seen in pulmonary SS, especially in follicular bronchiolitis ${ }^{35}$.

Certain viruses have also been implicated in the pathogenesis of SS due to their tropism for salivary and lacrimal glands, such as the Herpesviridae family and retroviruses ${ }^{36}$. The main mechanism that explain the association of viral infection and autoimmune disease are molecular mimicry, polyclonal lymphocyte proliferation and inflammation leading to increased immunogenicity of epithelium ${ }^{37}$.

\section{Epidemiology}

Studies have identified various risk factors predisposing patients with SS to develop pulmonary manifestations, these include male gender, tobacco use, old age, ANA positivity, late onset, and prolonged course of disease ${ }^{38,39}$. Pulmonary function tests (PFTs) serve as an important tool in identifying pattern of disease. In the presence of ILD a restrictive pattern is seen with a reduction in total lung capacity (TLC), forced vital capacity (FVC), forced expiratory volume in 1s (FEV1) and normal or increased FEV1/FVC ratio. On the contrary, involvement of airways can also manifest as an obstructive pattern on PFTs. Of note, decreased diffusion capacity of lung for carbon monoxide (DLCO) can be seen in both patterns. In the presence of concomitant restrictive/ obstructive lung disease involvement PFTS may appear to be preserved ${ }^{40-42}$.

As established above, ILD is the most common pulmonary manifestation of SS and is demonstrated in different forms. A study involving 225 patients with SS, identified 201 patients with ILD as per HRCT findings out of which non-specific interstitial pneumonia (NSIP) was the most commonly identified pattern seen in $45.5 \%$ of patients followed by usual interstitial pneumonia (10.1\%), lymphocytic interstitial pneumonia (8.2\%), bronchiolitis (7.6\%), organizing pneumonia (3.8\%) and unclassified ILD pattern in $24.7 \%{ }^{38}$.

\section{Nonspecific Interstitial Pneumonia (NSIP)}

NSIP is one of the most frequently encountered presentations of ILD in $\mathrm{SS}^{43}$. It is further stratified into cellular (cNSIP) and fibrotic NSIP (fNSIP). Comparatively, fNSIP is associated with poorer prognosis. Histologically it appears as homogenous fibrosis of pulmonary interstitium without prominent architectural disruption or presence of honeycombing pattern. Imaging may show ground glass opacities and bi-basilar reticular infiltrates, traction bronchiectasis and pulmonary consolidation with subpleural sparing ${ }^{44}$. Overall, symmetric, and basilar opacities, sub-pleural sparing in the absence of honeycombing should allow differentiation between NSIP and UIP.
NSIP can eventually lead to spontaneous resolution with risk of recurrence of disease, stable irreversible disease or progressively worsening of disease despite adequate medical therapy ${ }^{45}$.

\section{Usual Interstitial Pneumonia (UIP)}

In contrast to NSIP, the hallmark of UIP is the presence of honeycombing pattern with/without airspace enlargement and parenchymal distortion. Although uncommon in SS, features of UIP include patchy areas of dense fibrosis and lung destruction alternating with normal lung parenchyma and lesions present along the peripheral lung lobes and sub-pleural regions ${ }^{46}$. UIP is present in $\sim 16 \%$ patients with $\mathrm{SS}^{43}$. This pattern is prevalent among patients with onset of disease at an older age and is associated with poorer prognosis despite therapy ${ }^{23}$.

\section{Lymphocytic Interstitial Pneumonia (LIP)}

LIP was the first described pulmonary manifestation of SS in $1973^{47}$. Although, it is also known as a pre-neoplastic condition, malignant conversion in SS is considered unusual $^{48}$.

Physical examination is characterized by shortness of breath, cough, and bilateral inspiratory crackles. PFTs demonstrate a restrictive pattern and imaging is consistent with thickening of broncho-vascular bundles. On histology, interstitial infiltrate comprising of lymphocytes, polyclonal plasma cells and histiocytes invading the alveolar septa is seen. Ground glass opacities are a common finding in the HRCT and appear to correlate with the degree of inflammation ${ }^{49}$. Biopsy is usually necessary to exclude presence of lymphoma or amyloidosis ${ }^{10,50}$.

Overall, LIP is a reversible condition with potential risk of progression. Most patients respond well to corticosteroids.

\section{Organizing Pneumonia (OP)}

Organizing pneumonia is a rare manifestation in SS and is more characteristically seen in rheumatoid arthritis. Patients usually present with fever and fatigue in addition to shortness of breath. Imaging is consistent with peripheral pulmonary opacities and subpleural involvement. Histology is characterized by infiltration of fibroblasts in the alveolar ducts and intraluminal spaces with extension to nearby airspaces ${ }^{13}$.

\section{How to differentiate between pulmonary manifestations of SS and other autoimmune conditions}

Systemic lupus erythematosus (SLE): Overall tissue injury in SLE is characterized by autoantibody production, immune complex formation and ultimately deposition in tissues. SLE can affect the pleura, pulmonary vasculature as well as the lung parenchyma. The most common 
manifestation seen is unilateral or bilateral pleural effusion, frequently associated with pericardial effusion, and pulmonary bacterial infections ${ }^{51}$. Pulmonary fibrosis is not typically seen in SLE, however, if present, fibrosis usually involves the peripheral lung parenchyma and lower lobes. Rare manifestations seen in SLE include acute lupus pneumonia and shrinking lung syndrome ${ }^{52}$. SLE and SS are closely related genotypically and phenotypically, targeting a similar population (mostly women), however, in the latter it is important to note presence of mucosal inflammation, xerostomia and xerotrachea, manifested as small airway disease ${ }^{9}$.

Rheumatoid arthritis: Pulmonary manifestation of RA usually present within the first 5 years of disease and can be secondary to either disease progression or effect of immune-modulating drugs used to treat RA such as methotrexate ${ }^{53}$. ILD is the most common pulmonary manifestation of RA and risk factors include male gender, 5 th to 6 th decade of life, prolonged disease course, smoking and higher level of rheumatoid factor. Usual Interstitial Pneumonia (UIP) is the most identified pattern followed by NSIP, OP, DIP, LIP, and acute interstitial pneumonia. UIP in RA is associated with fewer fibroblast foci, a higher number of germinal centers histologically and a greater 5 -year mortality rate ${ }^{54}$. Treatment is recommended with anti-inflammatory and/or immunomodulatory drugs, mainly glucocorticoids, cyclophosphamide, mycophenolate mofetil and azathioprine ${ }^{55,56}$. Novel therapies are emerging, such as nintedanib in patients with connective tissue disease (CTD) related progressive form of $\operatorname{ILD}^{57,58}$.

Other manifestations in RA include pleural effusion, rheumatoid nodules involving vocal cords, arthritis of cricoarytenoid joint, recurrent laryngeal/vagus nerve vasculitis, pulmonary nodules, bronchiolitis and bronchiectasis ${ }^{59}$. Presence of pulmonary nodules and small airway disease overlaps between the presentation of SS and RA.

Systematic sclerosis: Systemic sclerosis (SSc) involves vascular inflammation and tissue fibrosis ${ }^{60}$. The fibrotic extension may involve several tissues and organs, frequently involving the lungs ${ }^{61}$. Pulmonary compromise is associated with increased morbidity and mortality, especially in the presence of pulmonary arterial hypertension (PAH) and ILD ${ }^{62,63}$. therefore, early detection and initiation of treatment is crucial to improve survival in these patients ${ }^{63,64}$. ILD usually presents within the first 3-5 years of diagnosis ${ }^{61}$, and is characterized by a restrictive pattern on PFTs and imaging (HRCT) is consistent with reticulation and ground glass opacities mainly in bilateral basilar pulmonary lobes ${ }^{65}$. Like SS, the typical pattern is NSIP.

$\mathrm{PAH}$ is frequently becoming a common presentation of pSS, requiring more attention at the time of diagnosis and subsequent follow-ups. It is important to differentiate PAH associated with SS and SSc, some important diagnostic identifiers are presence of pericardial effusion, hepatic injury, Raynaud's phenomenon, and positive rheumatoid factor which are typically seen in SSc only ${ }^{66,67}$.

\section{Treatment}

Pulmonary manifestations of SS have an unpredictable course of evolution, therefore, randomized clinical trials are limited. Standard treatment involves a multi-disciplinary approach, involving pulmonology, rheumatology, radiology, cardiology, and rehabilitative services to provide a multimodal treatment approach.

For asymptomatic patients with mild findings of ILD on imaging, a passive approach requiring routine follow up and assessment with repeat imaging at an interval of 6 months to 1 year can be adopted. For mild symptoms of bronchitis, isotonic saline irrigation, nebulized hypertonic saline and bronchodilators can be used ${ }^{6}$.

For severe or progressive interstitial lung disease, multiple drugs have been studied to assess efficacy. The standard empiric therapy is use of glucocorticoids at an initial dose of $0.5-1 \mathrm{mg} / \mathrm{kg}$ daily, gradually tapered ${ }^{68}$. Immunosuppressive drugs such as cyclophosphamide, azathioprine, and mycophenolate mofetil can be added either as first line therapy or maintenance therapy to reduce steroid dependence, as per clinical trials. Overall, studies performed to determine treatment strategies for connective tissue disease associated ILD (majority associated with systemic sclerosis) and NSIP have demonstrated maximal efficacy with cyclophosphamide followed by mycophenolate mofetil ${ }^{69,70}$. Other treatment options such as Rituximab which targets B-cells is well known for its efficacy regarding systemic manifestation of SS such as vasculitis and arthritis, however, its efficacy has only been studied in small groups of patients ${ }^{71}$. Abatacept, CTLA-4 monoclonal antibody that competitively blocks action of T-cells, has also shown treatment benefit in cases.

Another potential treatment aspect is targeting interleukins, IFN-receptors and signaling pathways. Many studies are exploring the potential role of FDA approved drugs which are routinely used for management of rheumatoid arthritis, systemic lupus erythematosus, and ulcerative colitis, in improving pulmonary manifestations of $\mathrm{SS}^{72,73}$.

Further large-scale studies with longer follow-up periods are required to determine the overall efficacy of treatment options to develop treatment guidelines.

\section{Conclusions}

Sjögren's syndrome is an autoimmune connective tissue disease mainly targeting the salivary and lacrimal glands. In a subgroup of SS patients, the disease will 
extend to extra-glandular organs and tissues amongst which the pulmonary system is one of the most frequently involved system. NSIP pattern is the most common type of parenchymal inflammation. The pathogenesis is complex involving the innate and acquired immune systems. Different inflammatory cytokine pathways and exogenous antigen such as viruses are implicated in the pathogenesis.

Therapy should focus on the type of compartment involved. Although, there are no major trials regarding effective treatment modalities, the mainstay of therapy is a combination of treatment modalities targeting airway inflammation and systemic manifestations. Concurrent usage of systemic glucocorticoids and immune modulators / suppressants appears to be the most effective treatment option when the functional capacities are compromised. The use of biological therapy such as Rituximab has shown potential in small case series. Further studies evaluating therapeutic options for SS are eagerly awaited.

\section{References}

1. Moutsopoulos HM, Chused TM, Mann DL, et al. Sjogren's syndrome (Sicca syndrome): current issues. Ann Intern Med. 1980; 92(2 Pt 1): 212-26.

2. Omdal R, Mellgren SI, Norheim KB. Pain and fatigue in primary Sjogren's syndrome. Rheumatology (Oxford). 2019.

3. Fox RI. Sjogren's syndrome. Lancet. 2005; 366(9482): 321-31.

4. Vitali C, Bombardieri S, Jonsson R, et al. Classification criteria for Sjogren's syndrome: a revised version of the European criteria proposed by the American-European Consensus Group. Ann Rheum Dis. 2002 ; 61(6): 554-8.

5. Shiboski CH, Shiboski SC, Seror R, et al. 2016 American College of Rheumatology/European League Against Rheumatism Classification Criteria for Primary Sjogren's Syndrome: A Consensus and DataDriven Methodology Involving Three International Patient Cohorts. Arthritis Rheumatol. 2017; 69(1): 35-45.

6. Gupta S, Ferrada MA, Hasni SA. Pulmonary Manifestations of Primary Sjogren's Syndrome: Underlying Immunological Mechanisms, Clinical Presentation, and Management. Front Immunol. 2019; 10: 1327.

7. Uffmann $M$, Kiener HP, Bankier AA, et al. Lung manifestation in asymptomatic patients with primary Sjogren syndrome: assessment with high resolution CT and pulmonary function tests. J Thorac Imaging. 2001; 16(4): 282-9.

8. Nannini C, Jebakumar AJ, Crowson CS, et al. Primary Sjogren's syndrome 1976-2005 and associated interstitial lung disease: a population-based study of incidence and mortality. BMJ Open. 2013; 3(11): e003569.

9. Papiris SA, Maniati M, Constantopoulos SH, et al. Lung involvement in primary Sjogren's syndrome is mainly related to the small airway disease. Ann Rheum Dis. 1999; 58(1): 61-4.

10. Flament $\mathrm{T}$, Bigot A, Chaigne B, et al. Pulmonary manifestations of Sjogren's syndrome. Eur Respir Rev. 2016; 25(140): 110-23.

11. Papiris SA, Saetta M, Turato G, et al. CD4-positive T-lymphocytes infiltrate the bronchial mucosa of patients with Sjogren's syndrome. Am J Respir Crit Care Med. 1997; 156(2 Pt 1): 637-41.

12. Moutsopoulos HM. Sjogren's syndrome: a forty-year scientific journey. J Autoimmun. 2014; 51: 1-9.
13. Stojan G, Baer AN, Danoff SK. Pulmonary manifestations of Sjogren's syndrome. Curr Allergy Asthma Rep. 2013; 13(4): 354-60.

14. Lopez Velazquez M, Highland KB. Pulmonary manifestations of systemic lupus erythematosus and Sjogren's syndrome. Curr Opin Rheumatol. 2018; 30(5): 449-64.

15. Di Stefano A, Turato G, Maestrelli P, et al. Airflow limitation in chronic bronchitis is associated with T-lymphocyte and macrophage infiltration of the bronchial mucosa. Am J Respir Crit Care Med. 1996; 153(2): 629-32.

16. Soto-Cardenas MJ, Perez-De-Lis M, Bove A, et al. Bronchiectasis in primary Sjogren's syndrome: prevalence and clinical significance. Clin Exp Rheumatol. 2010; 28(5): 647-53.

17. Stålenheim G, Gudbjörnsson B. Anti-inflammatory drugs do not alleviate bronchial hyperreactivity in Sjögren's syndrome. ALLERGY. 1997; 52(4): 423-7.

18. Ludviksdottir D, Janson C, Bjornsson E, et al. Different airway responsiveness profiles in atopic asthma, nonatopic asthma, and Sjogren's syndrome. BHR Study Group. Bronchial hyperresponsiveness. Allergy. 2000; 55(3): 259-65.

19. Yousem SA, Colby TV, Carrington CB. Follicular bronchitis/ bronchiolitis. Hum Pathol. 1985; 16(7): 700-6.

20. Matsuyama N, Ashizawa K, Okimoto T, et al. Pulmonary lesions associated with Sjogren's syndrome: radiographic and CT findings. Br J Radiol. 2003; 76(912): 880-4.

21. Borie R, Schneider S, Debray MP, et al. Severe chronic bronchiolitis as the presenting feature of primary Sjogren's syndrome. Respir Med. 2011; 105(1): 130-6.

22. Luppi F, Sebastiani M, Silva M, et al. Interstitial lung disease in Sjögren's syndrome: a clinical review. Clin Exp Rheumatol. 2020; 38(126): S291-S300.

23. Ting Zhang FY, Li Xu, Wenjia Sun, et al. Characteristics of patients with primary Sjögren's syndrome associated interstitial lung disease and relevant features of disease progression. Clinical Rheumatology. 2020; 39: 1561-8.

24. Zhang T, Yuan F, Xu L, et al. Characteristics of patients with primary Sjogren's syndrome associated interstitial lung disease and relevant features of disease progression. Clin Rheumatol. 2020; 39(5): 1561-8.

25. Daniels TE, Cox D, Shiboski CH, et al. Associations between salivary gland histopathologic diagnoses and phenotypic features of Sjogren's syndrome among 1,726 registry participants. Arthritis Rheum. 2011; 63(7): 2021-30.

26. Moutsopoulos HM. Sjögren's syndrome: autoimmune epithelitis. Clin Immunol Immunopathol. 1994; 72(2): 162-5.

27. Katsiougiannis S, Tenta R, Skopouli FN. Skopouli. Autoimmune epithelitis (Sjögren's syndrome); the impact of metabolic status of glandular epithelial cells on auto-immunogenicity. Journal of Autoimmunity. 2019; 104: 102335.

28. Kakugawa T, Sakamoto N, Ishimoto H, et al. Lymphocytic focus score is positively related to airway and interstitial lung diseases in primary Sjogren's syndrome. Respir Med. 2018; 137: 95-102.

29. Sandhya P, Kurien BT, Danda D, et al. Update on Pathogenesis of Sjogren's Syndrome. Curr Rheumatol Rev. 2017; 13(1): 5-22.

30. Wang F, Jackson MW, Maughan V, et al. Passive transfer of Sjogren's syndrome IgG produces the pathophysiology of overactive bladder. Arthritis Rheum. 2004; 50(11): 3637-45.

31. Kawakami A, Nakashima K, Tamai M, et al. Toll-like Receptor in Salivary Glands from Patients with Sjögren's Syndrome: Functional Analysis by Human Salivary Gland Cell Line. Journal of Rheumatology. 2007: 34(5): 1019-26. 
32. McGrath-Morrow S, Laube B, Tzou SC, et al. IL-12 overexpression in mice as a model for Sjogren lung disease. Am J Physiol Lung Cell Mol Physiol. 2006; 291(4): L837-46.

33. Verstappen GM, Corneth OBJ, Bootsma H, et al. Th17 cells in primary Sjogren's syndrome: Pathogenicity and plasticity. J Autoimmun. 2018; 87: $16-25$.

34. Milovanovic J, Arsenijevic A, Stojanovic B, et al. Interleukin-17 in Chronic Inflammatory Neurological Diseases. Front Immunol. 2020; 11: 947.

35. Salomonsson S, Jonsson MV, Skarstein K, et al. Cellular basis of ectopic germinal center formation and autoantibody production in the target organ of patients with Sjogren's syndrome. Arthritis Rheum. 2003; 48(11): 3187-201.

36. Igoe A, Scofield RH. Autoimmunity and infection in Sjogren's syndrome. Curr Opin Rheumatol. 2013; 25(4): 480-7.

37. Jean-FrançoisBach. Infections and autoimmune diseases. Journal of autoimmunity. 2005; 25: 74-80.

38. Wang Y, Hou Z, Qiu M, et al. Risk factors for primary Sjogren syndromeassociated interstitial lung disease. J Thorac Dis. 2018; 10(4): 210817.

39. García-Carrasco M, Ramos-Casals M, Rosas J, et al. Primary Sjögren Syndrome Clinical and Immunologic Disease Patterns in a Cohort of 400 Patients. Medicine (Baltimore). 2002; 81(4): 270-80.

40. Luppi F, Sebastiani M, Sverzellati N, et al. Lung complications of Sjogren syndrome. Eur Respir Rev. 2020; 29(157): :200021.

41. Palm O, Garen T, Berge Enger T, et al. Clinical pulmonary involvement in primary Sjogren's syndrome: prevalence, quality of life and mortality-a retrospective study based on registry data. Rheumatology (Oxford). 2013 ;52(1): 173-9.

42. Reina D, Roig Vilaseca D, Torrente-Segarra V, et al. Sjogren's syndromeassociated interstitial lung disease: A multicenter study. Reumatol Clin. 2016; 12(4): 201-5.

43. Ramos-Casals M, Brito-Zeron P, Seror R, et al. Characterization of systemic disease in primary Sjogren's syndrome: EULAR-SS Task Force recommendations for articular, cutaneous, pulmonary and renal involvements. Rheumatology (Oxford). 2015; 54(12): 2230-8.

44. Elicker B, Pereira CA, Webb R, et al. High-resolution computed tomography patterns of diffuse interstitial lung disease with clinical and pathological correlation. J Bras Pneumol. 2008; 34(9): 715-44.

45. Travis WD, Costabel U, Hansell DM, et al. An official American Thoracic Society/European Respiratory Society statement: Update of the international multidisciplinary classification of the idiopathic interstitial pneumonias. Am J Respir Crit Care Med. 2013; 188(6): 733-48.

46. Enomoto Y, Takemura T, Hagiwara E, et al. Features of usual interstitial pneumonia in patients with primary Sjogrens syndrome compared with idiopathic pulmonary fibrosis. Respir Investig. 2014; 52(4): 227 35 .

47. Liebow AA, Carrington CB. Diffuse pulmonary lymphoreticular infiltrations associated with dysproteinemia. Medical Clinics of North America. 1973; 57(3): 809-43.

48. Cha SI, Fessler MB, Cool CD, et al. Lymphoid interstitial pneumonia: clinical features, associations and prognosis. Eur Respir J. 2006; 28(2): 364-9.

49. Dong X, Gao YL, Lu Y, et al. Characteristics of primary Sjogren's syndrome related lymphocytic interstitial pneumonia. Clin Rheumatol. 2021; 40(2): 601-12.

50. Arcadu A, Moua T, Yi ES, et al. Lymphoid Interstitial Pneumonia and Other Benign Lymphoid Disorders. Semin Respir Crit Care Med. 2016; 37(3): 406-20.
51. Pego-Reigosa JM, Medeiros DA, Isenberg DA. Respiratory manifestations of systemic lupus erythematosus: old and new concepts. Best Practice \& Research Clinical Rheumatology. 2009; 23(4): 469-80.

52. Cojocaru M, Cojocaru IM, Silosi I, et al. Pulmonary Manifestations of Systemic Autoimmune Diseases. A journal of clinical medicine. 2011; 6(3): 224-9.

53. Marigliano B, Soriano A, Margiotta D, et al. Lung involvement in connective tissue diseases: a comprehensive review and a focus on rheumatoid arthritis. Autoimmun Rev. 2013; 12(11): 1076-84.

54. Kim EJ, Collard HR, King TE Jr. Rheumatoid arthritis-associated interstitial lung disease: the relevance of histopathologic and radiographic pattern. Chest. 2009; 136(5): 1397-405.

55. Roca F, Dominique S, Schmidt J, et al. Interstitial lung disease in primary Sjogren's syndrome. Autoimmun Rev. 2017; 16(1): 48-54.

56. Parambil JG, Myers JL, Lindell RM, et al. Interstitial lung disease in primary Sjogren syndrome. Chest. 2006; 130(5): 1489-95.

57. Richeldi L, Costabel U, Selman M, et al. Efficacy of a tyrosine kinase inhibitor in idiopathic pulmonary fibrosis. N Engl J Med. 2011; 365(12): 1079-87.

58. Richeldi L, du Bois RM, Raghu G, et al. Efficacy and safety of nintedanib in idiopathic pulmonary fibrosis. N Engl J Med. 2014; 370(22): 207182.

59. Shaw M, Collins BF, Ho LA, et al. Rheumatoid arthritis-associated lung disease. Eur Respir Rev. 2015; 24(135): 1-16.

60. Denton CP, Khanna D. Systemic sclerosis. Lancet. 2017; 390(10103): 1685-99.

61. Caron M, Hoa S, Hudson M, et al. Pulmonary function tests as outcomes for systemic sclerosis interstitial lung disease. Eur Respir Rev. 2018; 27(148): 170102.

62. Elhai M, Meune C, Avouac J, et al. Trends in mortality in patients with systemic sclerosis over 40 years: a systematic review and metaanalysis of cohort studies. Rheumatology (Oxford). 2012; 51(6): 1017-26.

63. Steen VD, Medsger TA. Changes in causes of death in systemic sclerosis, 1972-2002. Ann Rheum Dis. 2007; 66(7): 940-4.

64. Hoffmann-Vold AM, Aalokken TM, Lund MB, et al. Predictive value of serial high-resolution computed tomography analyses and concurrent lung function tests in systemic sclerosis. Arthritis Rheumatol. 2015; 67(8): 2205-12.

65. Hassoun PM. Lung involvement in systemic sclerosis. Presse Med. 2011; 40(1 Pt 2): e3-e17.

66. Liu Z, Yang X, Tian Z, et al. The prognosis of pulmonary arterial hypertension associated with primary Sjogren's syndrome: a cohort study. Lupus. 2018; 27(7): 1072-80.

67. Yan S, Li M, Wang H, et al. Characteristics and risk factors of pulmonary arterial hypertension in patients with primary Sjogren's syndrome. Int J Rheum Dis. 2018; 21(5): 1068-75.

68. Parambil JG, Myers JL, Lindell RM, et al. Interstitial lung disease in primary Sjögren syndrome. Chest. 2006; 130(5): 1489-95.

69. Fischer A, Brown KK, Du Bois RM, et al. Mycophenolate mofetil improves lung function in connective tissue disease-associated interstitial lung disease. J Rheumatol. 2013; 40(5): 640-6.

70. Saraux A, Pers JO, Devauchelle-Pensec V. Treatment of primary Sjogren syndrome. Nature Reviews Rheumatology. 2016; 12(8): 456-71.

71. Chen MH, Chen CK, Chou HP, et al. Rituximab therapy in primary Sjögren's syndrome with interstitial lung disease: a retrospective cohort study. Clin Exp Rheumatology. 2016: 34(6): 1077-1084. 
72. Barnes PJ. Kinases as Novel Therapeutic Targets in Asthma and Chronic Obstructive Pulmonary Disease. Pharmacol Rev. 2016; 68(3): 788-815.
73. Furie R, Khamashta M, Merrill JT, et al. Anifrolumab, an Anti-Interferonalpha Receptor Monoclonal Antibody, in Moderate-to-Severe Systemic Lupus Erythematosus. Arthritis Rheumatol. 2017; 69(2): 376-86. 